\title{
IDENTIFICATION OF JET INSTABILITY WAVES AND DESIGN OF A MICROPHONE ARRAY
}

\author{
Takao Suzuki and Tim Colonius ${ }^{\dagger}$ \\ Division of Engineering and Applied Science \\ California Institute of Technology, Pasadena, California 91125
}

\begin{abstract}
We propose a diagnostic technique to detect instability waves in a round jet using a conical microphone array. The detection algorithm is analogous to the beam-forming technique, which is used with a far-field microphone array to localize noise sources. By replacing the reference solutions used in the conventional beam-forming with eigenfunctions from a linear stability analysis, the amplitude of the low-frequency azimuthal instability waves can be inferred. We use a DNS database and experimental measurements of the mean flow to design a phased microphone array which is placed just outside the shear layer near the nozzle exit. We validate the technique in experiments by checking for consistency of the radial decay, phase correlation, and streamwise evolution of the hydrodynamic pressure. The comparison between the experiment and linear stability analysis shows good agreement, particularly near the most amplified frequency of each azimuthal mode.
\end{abstract}

\section{INTRODUCTION}

Passive mixing devices such as tabs and chevrons lead to jet noise reduction at low frequencies (typically including the peak frequency), but enhance noise levels at higher frequencies $[8,24,28,29,32]$. Active control devices such as piezoelectric flaps and microjets are presently being examined by a number of groups (e.g. Refs. $[1,7]$ ). For either case noise reduction efforts involve extensive model and full-scale testing - as of now there is no reliable model available to connect particular devices with the far field sound level. One way in which mixing devices are thought to effect the sound field is by altering the mean flow and stability characteristics of the initial

Copyright (C) 2004 by Takao Suzuki and Tim Colonius. Published by the American Institute of Aeronautics and Astronautics, Inc., with permission. shear layers and, in turn, the large-scale flow structures that are connected to the low frequency noise.

Large-scale structures are related to linear instabilities of the time-averaged jet flow field. The relation between the instability waves and the acoustic field, on the other hand, has been a topic of debate for many years. Quantitative models for the near field evolution have been developed $[19,20,22]$. When instability waves convect with supersonic speed (relative to ambient), they give rise to strong Mach wave radiation that can be predicted using the method of the matched asymptotic expansions [31] or with the parabolized stability equations (PSE), (e.g. Ref. [5]). However, the noise from linear, subsonically convecting instability waves is much weaker and depends very sensitively on the precise structure of the wave packet as well as nonlinear interactions $[14,21]$. Therefore, in the subsonic case, only qualitative models of the sound generation from instability waves have been deduced $[11,19]$. Further modeling developments, especially utilizing the nonlinear PSE method [9] are being developed. A potential difficulty with all instability wave models is that their initial amplitudes near the nozzle are uncertain.

The aim of the present research is to develop an experimental technique which identifies the signatures and amplitudes of instability waves convecting in the jet. This identification technique may provide useful input for the design of mixing devices and modeling efforts in three ways. First, it would provide an initial disturbance field for the unstable modes that could be fed to the models discussed above. Second, it would show how the mixing devices affect the development of instability waves in the jet. Finally, it can be used to correlate measured far-field sound with the near field behavior (see, for example, Ref. [27]).

The measurement technique we develop is based on previous non-intrusive diagnostic approaches for noise source identification with phased microphone arrays. These techniques, called 'beam-forming,' 
have successfully identified noise sources in many applications $[13,15,23,25,30,34]$. The basic idea is to detect position and amplitude of an acoustic source which best matches the reference solution (a monopole source being exclusively used) in a least square sense (c.f. Refs. $[12,17]$ ). We show here that a phased-microphone array placed just outside the shear layer (referred to as the 'hydrodynamic array') is indeed capable of detecting non-radiating waves, i.e. instability waves.

The key idea is to replace the monopole solution in the conventional beam-forming by an eigenfunction (or a field of eigenfunctions) obtained from a linear stability analysis. Outside the mixing layer, where nonlinear interaction is insignificant, there exists a region where the pressure fields associated with linear hydrodynamic waves (subsonically convecting) are exponentially decaying with radius from the jet axis (see figure 1). At still larger distances, acoustic disturbances, which are algebraically decaying, dominate the pressure fields. We place a conical array in this "linear hydrodynamic" region to effectively extract instability waves. To take into account the spreading effects of the mixing layer, we include the variation of eigenfunctions in the streamwise direction. Targeting the peak frequency of the far field sound $(S t \sim 0.3)$, we try to detect instability waves of the first three azimuthal modes $(m=0,1$, and 2$)$.

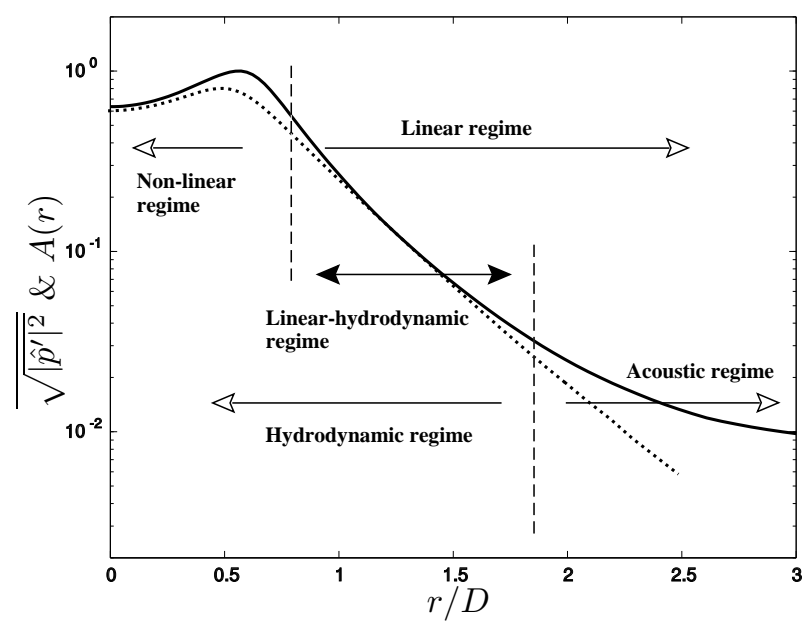

Figure 1: Diagram of the flow regimes of a round jet in the radial direction. The solid line depicts the time-averaged pressure fluctuation of the axisymmetric mode (i.e. $m=0$ ) at $x / D=4$ from DNS, and the dotted line denotes the corresponding eigenfunction calculated from a linear stability analysis. The maximum pressure fluctuation is normalized as unity and the eigenfunction is arbitrarily scaled.
We mention in passing that other diagnostic techniques have been used (or are presently being developed) to capture the large-scale structures in jets. In particular, Arndt et al. [2] experimentally studied the streamwise evolution $(x / D \approx 0 \sim 2.5)$ of the large-scale flow structures based on the proper orthogonal decomposition (POD). They measured phased array pressure data by distributing microphones in a conical shape and reconstructed the first three azimuthal POD modes. Citriniti \& George [10] measured POD modes at a cross section $(x / D=3)$ of a jet. Bonnet et al. [3] investigated vortical structures in a jet based on the stochastic estimation technique as well as POD. But, in those approaches the flow field cannot be readily reconstructed in regions outside the measured points. Moreover, physical implications of 'modes' obtained from these reduced order models are not clear. Optical diagnostic techniques, such as Particle Image Velocimetry (PIV), can access inside the flow fields in a non-contact fashion, but only provide velocity fields as opposed to pressure fields, which are more relevant to instability waves.

In this paper we describe how the hydrodynamic array has been designed and tested. In the next section, we study pertinent results from linear stability analysis and describe the detection algorithm. Next we briefly examine application of the proposed methodology to a direct numerical simulation (DNS) database of a subsonic round jet by Freund [14]. We then modify the array design for laboratory scale jets $\left(R e \sim 10^{5}\right)$ based on mean-flow data taken by Bridges \& Wernet using a PIV technique [6]. Finally, the algorithm is tested under various operating conditions in experiments. The flow conditions covered in the experiment include a range of Mach numbers $\left(u_{\text {jet }} / a_{\infty}=0.35 \sim 0.9\right.$ for unheated jets, $0.5 \sim 1.59$ for heated jets), temperature ratios $\left(T_{\text {jet }} / T_{\infty}=0.85 \sim 2.70\right)$, and the nozzle types (i.e. straight versus chevron nozzles). We close by discussing the capabilities and limitations of the hydrodynamic array.

\section{DETECTION ALGORITHMS}

\section{LINEAR STABILITY ANALYSIS}

We first briefly review some salient results from a linear stability analysis of a weakly non-parallel mean flow. This provides eigenfunctions, i.e. instability waves, which are used as reference solutions for the proposed detection algorithm later.

We assume that the flow is compressible and inviscid. The ansatz for the instability waves is 


$$
\Pi\left(\omega, m, a_{s} ; x, r, \theta\right)=a_{s} A(\omega, m ; r) \mathrm{e}^{-\mathrm{i}(\omega t-k x-m \theta)},
$$

where $\Pi$ denotes logarithmic pressure (i.e. $\Pi \equiv$ $\gamma^{-1} \log \left(p / p_{\infty}\right), \gamma$ being the specific heat ratio and $p_{\infty}$ the ambient pressure), $m$ is the azimuthal mode number, and $A$ denotes the eigenfunction, which is arbitrarily normalized here. Note that the eigenfunctions of positive and negative azimuthal modes are exactly identical.

To solve eigenfunctions, we substitute (1) into the third order convective wave operator $[18,26]$ which is Fourier transformed as follows:

$$
\frac{d}{d r}\left(\frac{r}{n^{2}} \frac{d A}{d r}\right)+\left(n^{2}-k^{2}-\frac{m^{2}}{r^{2}}\right) \frac{r}{n^{2}} A=0,
$$

where $n(r) \equiv[\omega-k U(r)] / a(r), U(r)$ being the mean velocity in the axial direction (denoted by $x$ ) and $a(r)$ the mean speed of sound.

The structure of the eigenfunction outside the jet is of special relevance to this study. At a larger radial distance, the Sommerfeld radiation condition must be satisfied:

$$
r^{1 / 2}\left(\frac{d A}{d r}-\mathrm{i} \sqrt{n_{\infty}^{2}-k^{2}} A\right) \rightarrow 0 \quad \text { as } \quad r \rightarrow \infty,
$$

where $n_{\infty} \equiv n(r=\infty)$. Outside the jet, it is clear that the eigenfunction is exponentially decaying with radius whenever the phase speed is subsonic, i.e. $\omega / k<a_{\infty}$.

In addition, the asymptotic behavior of $A(\omega, m ; r)$ near the jet axis is given by

$$
A(\omega, m ; r) \sim r^{m} \quad \text { as } \quad r \rightarrow 0 .
$$

In this study, we are interested in families of the most unstable mode, i.e. the largest $-\operatorname{Im}[k]$, for each $m$. For typical velocity and temperature profiles of a subsonic round jet, at most only one family of unstable modes exists for each $m$. Figure 2 depicts growth rates of instability waves for the first three azimuthal modes $(m=0 \sim 2)$. Each family has a peak growth rate between $S t=0.2 \sim 0.4$ at a cross section specified here $(x / D=2.25)$. Since the growth rates of the modes higher than $m=2$ are much weaker than those of the lower modes, we investigate only up to $m=2$ in this study.

To take into account the spreading effects of the mean flow, an eigenfunction is calculated at each axial station, $x$, by assuming that the velocity and temperature profiles are locally parallel. The composite eigenfunction (called "eigen-solution" thereafter) then becomes

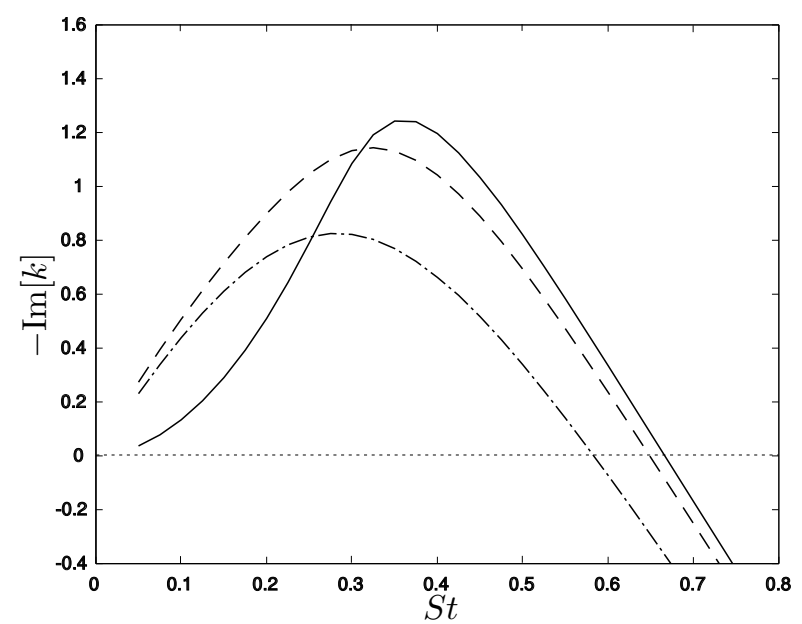

Figure 2: Growth rates of instability waves in a round jet $\left(M_{\text {jet }}=0.5\right)$. A spatial problem is solved based on a linear stability analysis, and the imaginary part of the wave-number is plotted: $m=0 ;---, m=1 ;-\cdots-\cdot-, m=2$. The mean velocity and temperature profiles were taken from the PIV data [6] at $x / D=2.25$.

$$
A(\omega, m ; x, r)=\bar{A}(\omega, m ; x, r) \exp \left[\mathrm{i} \int_{0}^{x} k(\xi) d \xi\right],
$$

where $\bar{A}(\omega, m ; x, r)$ is the normalized eigenfunction at each $x$ and $k(\xi)$ is the corresponding complex wave-number at $x=\xi$. We normalize $\bar{A}$ to be unity (with no imaginary part) at the radial position where the complex amplitude is maximum.

In computing the eigenfunctions, the velocity and temperature profiles are fitted with hyperbolic tangent profiles (either from DNS or experiment) at each cross section in a least square sense so that the equation can be smoothly integrated. A standard fourth order Runge-Kutta scheme was used to integrate the phase part of (5). Special care is taken to circumvent the critical layer [31], where eigen-modes are damped downstream as the mean flow spreads. Refer to an appendix for details.

Figure 3 compares the magnitude of eigenfunctions (5) with the averaged pressure fluctuations at $S t=0.3$ directly calculated from DNS [14]. The eigen-solutions capture the exponential growth and successive saturation in the streamwise direction, especially in $1.75<x / D<6.25$. The quantitative agreement is good for the $m=0$ and $m=1$ modes, while for $m=2$ the eigen-solution saturates earlier than the result from the DNS. The pressure fluctuation of the DNS has slower decay at a larger radius, 
at which sound waves become relevant. Near the mixing layer, on the other hand, vorticity and entropy fluctuations likely become significant (as observed in figure 1). It should be reminded that the Reynolds number in DNS is sufficiently low such that the mixing later is initially transitional.

We also compare the phase fields between eigenfunctions and pressure fluctuation from the DNS in figure 4. As discussed later, the beam-forming algorithm predicts amplitude based on phase matching. In the phase contours of pressure fluctuation from the DNS, several branch points appear (where multiple contours converge), at which the contribution from acoustic signals overcomes that from instability waves. Therefore, we must place the array closer to the centerline than these points. On the other hand, in the mixing layer we can observe some phase distortion, particularly in $m=0$ and $m=1$ near $x / D=3$, presumably due to nonlinear evolution. It is also important to notice that the agreement of phase variation in the hydrodynamic region, say $1<r / D<2$, is relatively good for the $m=0$ and $m=1$ modes, while there is some phase delay in the saturated region $(x / D>5)$ in $m=2$.

\section{BEAM-FORMING ALGORITHM}

In this section, we start with the existing beamforming algorithm (c.f. Refs. $[12,17]$ ) for acoustic source localization and subsequently extend it for instability wave identification in the following section. Suppose sound generated by a monopole source propagates in a free quiescent space. If we take a Fourier transform of pressure (or $\gamma^{-1} \log \left(p / p_{\infty}\right.$ ), as shown in (1)) in time, the pressure fluctuation at the point $\mathbf{x}$ may be represented by

$\hat{p}\left(\omega, a_{s}, \mathbf{x}_{s} ; \mathbf{x}\right)=\frac{1}{2} \frac{a_{s}(\omega)}{4 \pi\left|\mathbf{x}-\mathbf{x}_{s}\right|} \mathrm{e}^{\mathrm{i} k\left|\mathbf{x}-\mathbf{x}_{s}\right|} \equiv a_{s} \hat{P}\left(\mathbf{x}_{s} ; \mathbf{x}\right)$,

where $a_{s}$ denotes the complex source amplitude including an arbitrary phase shift, $\omega$ the angular frequency, $k$ the wave-number, $\mathbf{x}_{s}$ the source position, and $\hat{P}$ the form of a monopole solution. We consider $\hat{p}\left(\omega, a_{s}, \mathbf{x}_{s} ; \mathbf{x}\right)$ to be the reference solution for an acoustic source. On the other hand, suppose the pressure data are available at $N_{\text {mic }}$ observer points (i.e. microphones) and define the position of the $l^{\text {th }}$ observer to be $\mathbf{x}_{l}$. At each $\mathbf{x}_{l}$, we Fourier-transform the measured pressure data in time and denote them as $\hat{q}_{l}(\omega)$. Subsequently, we consider the square norm of the difference between the reference solution and the measured data and define the following cost function:
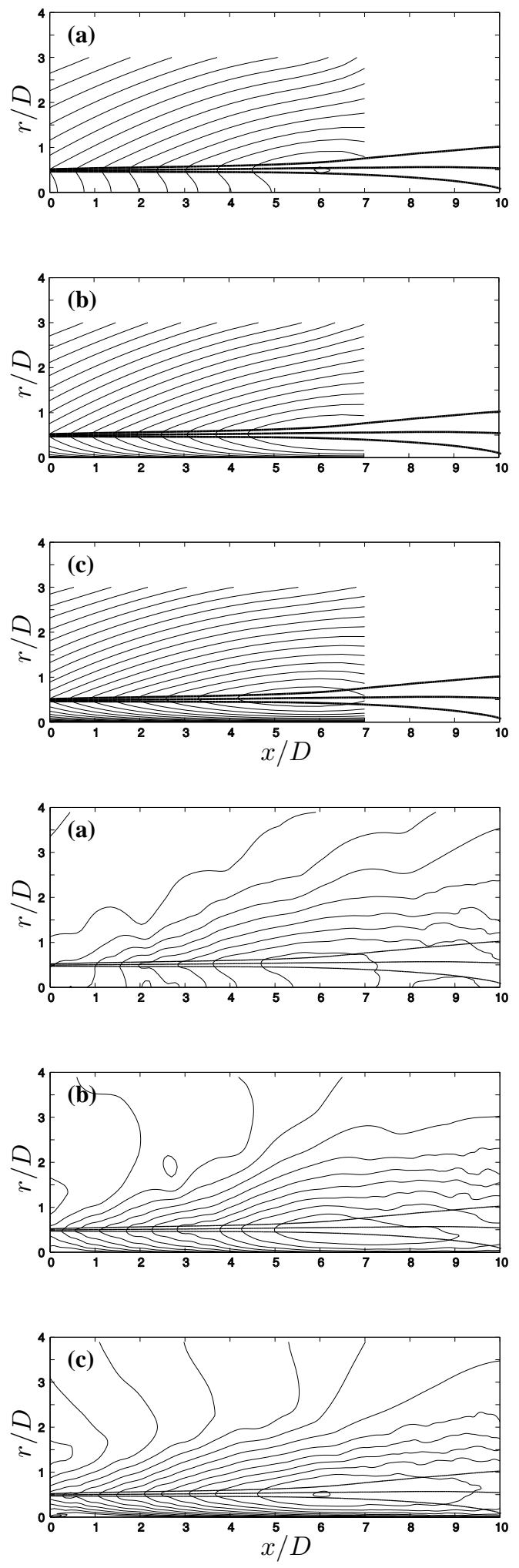

Figure 3: Contours of eigen-solutions (5) in pressure on the top three and averaged pressure fluctuation $\left(\overline{\sqrt{\left|\hat{p}^{\prime}\right|^{2}}}\right)$ from DNS on the bottom three: (a) $m=0$; (b) $m=1$; (c) $m=2$. A contour interval is $6 \mathrm{~dB}$ in both figures. Thicker lines depict iso-mean-velocity contours of $U_{\max } / 4, U_{\max } / 2$, and $3 U_{\max } / 4$. 

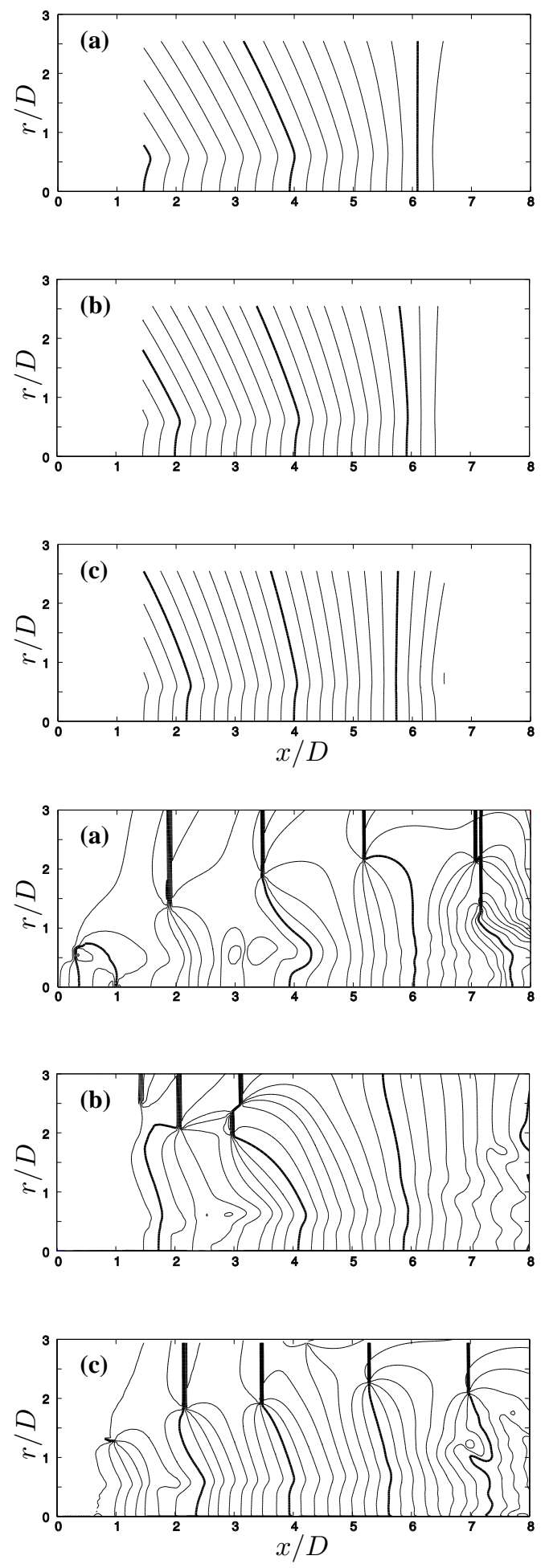

Figure 4: Iso-phase contours of the eigen-solutions on the top three and that of the pressure fluctuation from DNS on the bottom three. Notation is the same as figure 3. Contour interval is $45^{\circ}$, and the thicker lines denote every $360^{\circ}$.

$$
J\left(\omega, a_{s}, \mathbf{x}_{s}\right) \equiv \sum_{l=1}^{N_{\mathrm{mic}}}\left|\hat{p}\left(\omega, a_{s}, \mathbf{x}_{s} ; \mathbf{x}_{l}\right)-\hat{q}_{l}(\omega)\right|^{2} .
$$

Next, we determine the complex source amplitude, $a_{s}$, which optimizes the cost function above. By differentiating $J$ with respect to $a_{s}$, the source amplitude which minimizes (7) is given by

$$
\left(a_{s}\right)_{\min }\left(\omega, \mathbf{x}_{s}\right)=\frac{\sum_{l=1}^{N_{\text {mic }}} \hat{P}^{*}\left(\mathbf{x}_{s} ; \mathbf{x}_{l}\right) \hat{q}_{l}(\omega)}{\sum_{l=1}^{N_{\text {mic }}}\left|\hat{P}\left(\mathbf{x}_{s} ; \mathbf{x}_{l}\right)\right|^{2}},
$$

where the quantity with the superscript $*$ denotes complex conjugate. This quantity, (8), can be computed at each $\mathbf{x}_{s}$. Substituting (8) into (7), we obtain the minimized cost function as a function of $\omega$ and $\mathbf{x}_{s}$ :

$$
\begin{aligned}
J\left(\omega,\left(a_{s}\right)_{\min }, \mathbf{x}_{s}\right) & =\sum_{l=1}^{N_{\text {mic }}}\left|\hat{q}_{l}(\omega)\right|^{2} \\
& -\frac{\left|\sum_{l=1}^{N_{\text {mic }}} \hat{P}^{*}\left(\mathbf{x}_{s} ; \mathbf{x}_{l}\right) \hat{q}_{l}(\omega)\right|^{2}}{\sum_{l=1}^{N_{\text {mic }}}\left|\hat{P}\left(\mathbf{x}_{s} ; \mathbf{x}_{l}\right)\right|^{2}}
\end{aligned}
$$

Rearranging (9) and expressing it in a matrix form, we generally plot the following quantity to localize the acoustic source position:

$$
\begin{aligned}
Q\left(\omega, \mathbf{x}_{s}\right) & \equiv|\mathbf{q}|^{2}-J\left(\omega,\left(a_{s}\right)_{\min }\left(\omega, \mathbf{x}_{s}\right), \mathbf{x}_{s}\right) \\
& =\frac{\mathbf{P}^{*}}{|\mathbf{P}|} \cdot \mathbf{q q}^{*} \cdot \frac{\mathbf{P}}{|\mathbf{P}|}
\end{aligned}
$$

where $\mathbf{q} \equiv \hat{q}_{l}(\omega)$ and $\mathbf{P} \equiv \hat{P}\left(\mathbf{x}_{s} ; \mathbf{x}_{l}\right)$. The $l \times l$ matrix $\mathbf{q q}^{*}$ is called the "cross spectral matrix" and the vector $\mathbf{P} /|\mathbf{P}|$ (which has $l$ components) is the "steering vector." The position where the cost function becomes minimum, in turn, $Q\left(\omega, \mathbf{x}_{s}\right)$ becomes maximum, indicates the source position. At this point, $\left(a_{s}\right)_{\min }$ corresponds to the predicted complex source amplitude, and $Q\left(\omega, \mathbf{x}_{s}\right)$ to the source intensity. Thus, we can detect the position and amplitude of an acoustic source in a non-contact fashion.

\section{DETECTION OF INSTABILITY WAVES}

We extend the discussion above to detection of instability waves. We similarly assume that the pressure time histories are available at $N_{\text {mic }}$ observer points and define the transformed logarithmic pressure at the $l^{\text {th }}$ observer to be

$$
\hat{\Theta}_{l}(\omega) \equiv \frac{1}{2 \pi} \int_{0}^{\infty} \frac{1}{\gamma} \log \left[\frac{p\left(t, x_{l}, r_{l}, \theta_{l}\right)}{p_{\infty}}\right] \mathrm{e}^{\mathrm{i} \omega t} d t
$$


We also transform the eigenfunction (1) and define it as $\hat{\Pi}\left(\omega, m, a_{s} ; x_{l}, r_{l}, \theta_{l}\right)$. Subsequently, we introduce a cost function as

$$
J\left(\omega, m, a_{s}\right) \equiv \sum_{l=1}^{N_{\text {mic }}}\left|\hat{\Pi}\left(\omega, m, a_{s} ; x_{l}, r_{l}, \theta_{l}\right)-\hat{\Theta}_{l}(\omega)\right|^{2} .
$$

From (8), the amplitude of the instability waves, $a_{s}$, is then estimated as

$$
\begin{aligned}
& \left(a_{s}\right)_{\min }(\omega, m)= \\
& \frac{\sum_{l=1}^{N_{\text {mic }}} A^{*}\left(\omega, m ; r_{l}\right) \mathrm{e}^{-\operatorname{Im}[k] x_{l}} \mathrm{e}^{-\mathrm{i}\left(\operatorname{Re}[k] x_{l}+m \theta_{l}\right)} \hat{\Theta}_{l}(\omega)}{\sum_{l=1}^{N_{\text {mic }}}\left|A\left(\omega, m ; r_{l}\right)\right|^{2} \mathrm{e}^{-2 \operatorname{Im}[k] x_{l}}}
\end{aligned}
$$

for each $\omega$ and $m$. Thus, the magnitude and phase of instability waves for each $m$ can be computed with given $U(r)$ and $a(r)$. To be precise, the eigenfunctions are also functions of $x$ in this study, since we take into account the mean flow variation in the axial direction.

It should be mentioned that the principle idea of beam-forming is detection based on phase matching. As seen from (10), the quantity $Q\left(\omega, \mathbf{x}_{s}\right)$ is nearly maximized when the phases of the steering vector best match those of the cross spectral matrix. From the DNS data, the agreement in figure 4 demonstrates the validity of prediction based on phase matching, while the agreement in figure 3 ensures that the reference solution represents largescale coherent structures over a substantial region of a jet. Because the amplitude acts as a weight function, phase is most closely matched where the amplitude is highest. In detection of acoustic sources, variation in amplitude is generally insignificant provided that the array is reasonably far from the source relative to the aperture. By contrast, signals from instability waves exponentially grow in the axial direction; therefore, we must carefully distribute observer points so that amplitude variation between them is sufficiently small. This, in turn, helps reduce uncorrelated noise between observer points.

\section{DESIGN GUIDELINES FROM DNS}

To develop design procedures of the phased microphone array (i.e. hydrodynamic array) and evaluate the accuracy of the proposed detection method, the DNS database computed by Freund [14] for a round jet at a Mach number of $M=0.9$ and a Reynolds number of $R e=3600$ (based on the jet diameter) was used. The details of numerical procedures may be found in Ref. [14].
Using this DNS database, we construct reference solutions targeting at $S t=0.3$ as described above. We calculate the amplitude of each instability mode with the proposed beam-forming algorithm using every other point in the region indicated in figure 5 and treat this as the exact amplitude. Subsequently, we try to recover this amplitude from a limited number of microphones and evaluate the accuracy as a function of a microphone number and their distribution.

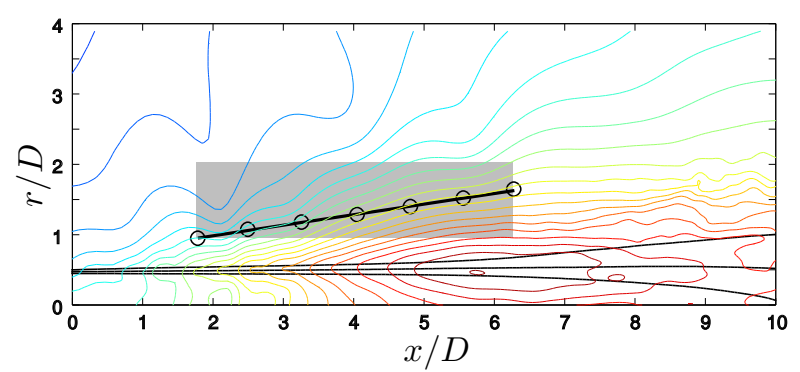

Figure 5: The domain where the amplitude of the reference solutions is calculated (denoted by a gray region), and the line along which the microphones are distributed (denoted by a solid line). As an example, seven microphones (i.e. rings) are distributed along the line. Contours of pressure fluctuations over $m=-9 \sim 9$ are superposed with an interval of $3 \mathrm{~dB}$.

The overall results suggest that the array requires at least seven rings in the streamwise direction and each ring needs six microphones (i.e. a total of 42 microphones) to resolve up to the $m=2$ mode. The azimuthal angles of the microphones are staggered in ever other ring (i.e. $30^{\circ}, 90^{\circ}, 150^{\circ}, 210^{\circ}, 270^{\circ}$, and $330^{\circ}$ for the first ring; $0^{\circ}, 60^{\circ}, 120^{\circ}, 180^{\circ}, 240^{\circ}$, and $300^{\circ}$ for the second ring, etc.) to suppress aliasing errors from higher azimuthal modes. The position of the array used with the DNS database is displayed in figure 5 . The accuracy of detection using this model array is estimated to be on the order of $30 \%(\sim 3$ $\mathrm{dB}$ ) for the overall pressure amplitude.

\section{EXPERIMENTAL FACILITIES}

\section{$\underline{\text { SHJAR }}$}

The noise measurement experiments using the Small Hot Jet Acoustic Rig (SHJAR) were conducted under the direction of Drs. Bridges, Lee, \& their co-workers at the NASA Glenn Research Center. A single-stream round jet was mounted about 2.5 meter high from the ground in an anechoic dome with a 20 meter radius. The jet exit was a simple 
converging nozzle (i.e. the maximum jet Mach number is unity), and the exit diameter of the jet was $5.08 \mathrm{~cm}$ (two inches). We tested three types of nozzle configurations: a straight nozzle, a six-lobe chevron nozzle with a penetration angle of $5^{\circ}$ (which is the same as the converging angle of the straight nozzle), and a six-lobe chevron nozzle with no-penetration (the chevrons are deflected outward relative to the straight nozzle). These nozzles are displayed in figure 6 .

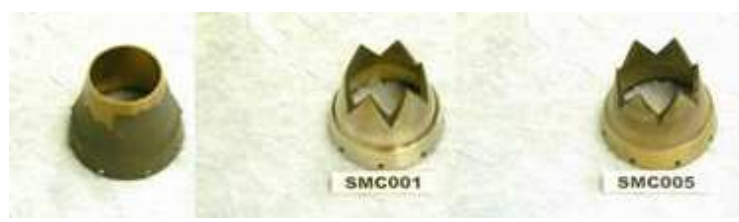

Figure 6: Photographs of the nozzles. Straight, chevrons with $5^{\circ}$ penetration, and chevrons with nopenetration from left to right.

We tested a variety of combinations of Mach numbers $\left(u_{\text {jet }} / a_{\infty}=0.35 \sim 0.9\right.$ for unheated cases, $0.5 \sim 1.59$ for heated cases), temperature ratios $\left(T_{\text {jet }} / T_{\infty}=0.85 \sim 2.7\right)$, and the nozzle types. These conditions of Mach numbers and temperature ratios followed the set points of the previous experiment by Tanna [33]. Here, we discuss only three representative cases of the straight nozzle focusing on validation aspects. Table 1 shows the flow conditions discussed in this paper. Note that the Reynolds number based on the jet diameter is approximately $0.5 \sim 1 \times 10^{5}$.

\begin{tabular}{|c|c|c|c|c|}
\hline Case & Set pt. & $M_{\text {jet }}$ & $T_{\text {jet }} / T_{\infty}$ & Nozzle \\
\hline A & 3 & 0.5 & $0.96($ cold $)$ & straight \\
\hline B & 7 & 0.9 & $0.85($ cold $)$ & straight \\
\hline C & 23 & 0.5 & 1.76 (hot) & straight \\
\hline
\end{tabular}

Table 1: Operating conditions of jet flows.

\section{MEAN FLOW DATA FROM PIV}

To construct the eigen-solutions for the experimental case, we used mean flow data measured by Bridges \& Wernet [6]. Three dimensional velocity fields were obtained using a PIV technique with resolutions of approximately $0.1 D$ and $0.02 D$ in the streamwise and the radial directions, respectively. The detailed procedures can be found in Ref. [6].

To smoothly integrate (2), we fitted the streamwise mean-velocity data from the PIV with a hyperbolic

tangent profile of $U(r) \approx \frac{U \max }{2}\left(\tanh \left[s\left(r+r_{0}\right)\right]-\tanh \left[s\left(r-r_{0}\right)\right]\right)$; namely, three parameters, $U_{\max }, s$, and $r_{0}$, were optimized in a least square sense at each cross section (see figure 7 for an example). From the streamwise velocity profiles, temperature profiles were computed using the Crocco-Busemann relation. The agreement with data obtained from pressure and temperature probes in a case at the highest temperature ratio is shown in figure 8 .

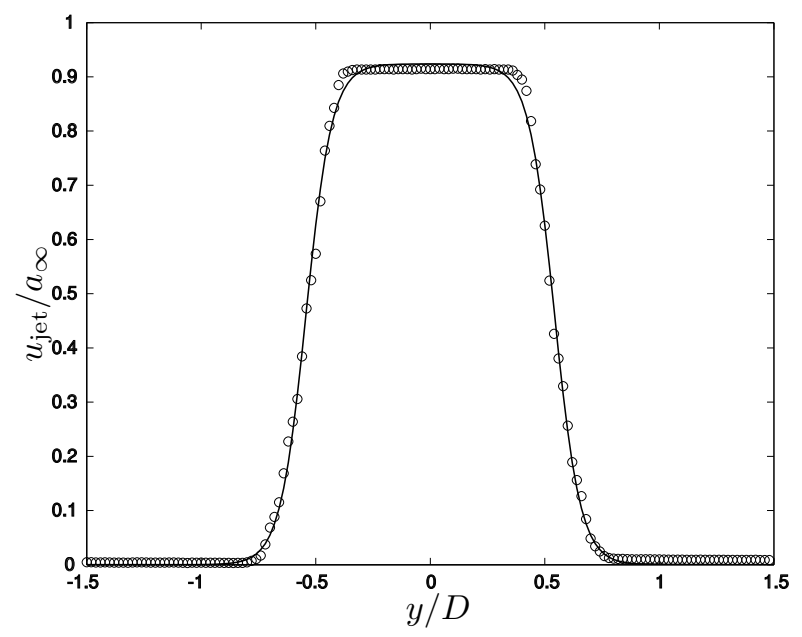

Figure 7: Velocity profile at $x / D=2$ (Case B): ०, data from the PIV; —_ approximate hyperbolictangent profile used for the linear stability analysis.

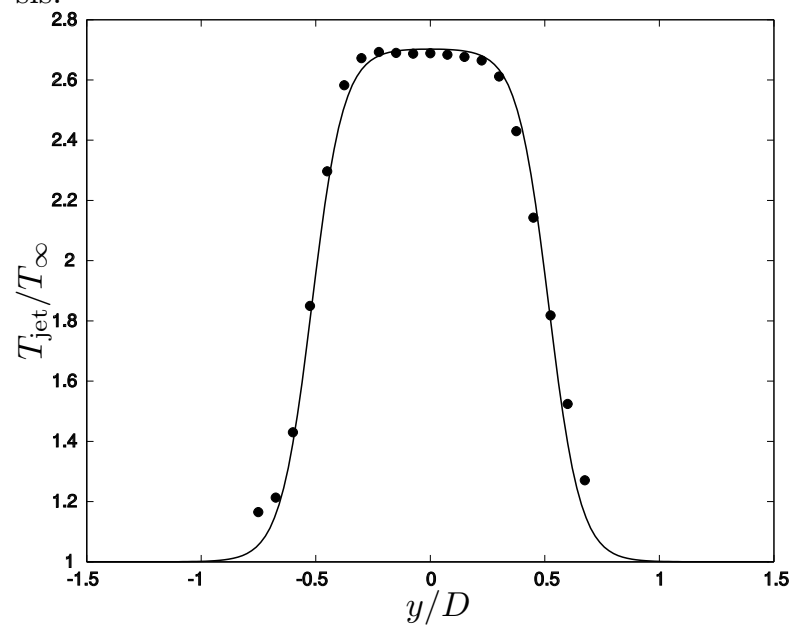

Figure 8: Temperature profile at $x / D=2$ $\left(u_{\text {jet }} / a_{\infty}=0.9\right.$ and $\left.T_{\text {jet }} / T_{\infty}=2.70\right): \circ$, data from direct probe measurement; —- approximate profile computed from the velocity profile using the Crocco-Busemann relation.

\section{HYDRODYNAMIC ARRAY}


Under laboratory conditions, the Reynolds number is at least one order of magnitude higher than that of the DNS, and the mixing layer of the jet spreads more rapidly. Therefore, starting with the overall requirements obtained from the preceding analyses with the DNS, we modify the configuration of the hydrodynamic array based on the PIV data mentioned above.

To determine the size of the array in the streamwise direction, we calculate wavelengths of instability waves based on the linear stability analysis for several frequencies and azimuthal modes (see table $2)$. We choose an unheated jet at $M_{\text {jet }} \equiv u_{\text {jet }} / a_{\infty}=$ 0.5 (Case A) as the baseline case for the design and use the mean velocity and temperature profiles at $x / D=2.25$, which is, in turn, approximately the center of the array in the streamwise direction.

Table 2 indicates that the array needs to extend as long as $5 D$ in order to cover two wavelengths of these modes at $S t=0.3$ (see $m=0$ in table 2). Hence, even if the array starts at $x / D=0$, it nearly reaches the end of the potential core, where the assumption of the locally-parallel mean flow fails (in addition, it is computationally more difficult to calculate eigenfunctions by a shooting method as the velocity profile becomes gentler downstream). On the other hand, we cannot locate microphones very close to the $x / D=0$ plane because of some interference near the nozzle exit. In particular, some modes associated internal aerodynamics become significant in unheated jets at higher Mach numbers (refer to an appendix). As a compromise, we set the array length to be $3.75 D$ starting approximately at $x / D=0.35$. This also satisfies a mechanical constraint regarding the microphone-ring spacing of $3.175 \mathrm{~cm}(0.625 \mathrm{D})$ in this experiment.

\begin{tabular}{|l|l|l|l|}
\hline & $m=0$ & $m=1$ & $m=2$ \\
\hline$S t=0.25$ & $3.30 D$ & $2.47 D$ & $2.13 D$ \\
\hline$S t=0.30$ & $2.47 D$ & $1.97 D$ & $1.75 D$ \\
\hline$S t=0.35$ & $1.87 D$ & $1.63 D$ & $1.49 D$ \\
\hline
\end{tabular}

Table 2: Wavelengths of instability waves obtained from the experimental velocity and temperature profiles for Case A at $x / D=2.25$.

The spreading angle of the array (the taper angle of a "cone") is set to be $11.3^{\circ}$. This is determined so that it is slightly steeper than the spreading angle of the mixing layer based on the velocity fluctuation from the PIV data. To test various radial positions of microphones, extra rings are equipped with the same spacings (the total number of the rings is 13). By shifting the whole array in the axial direction by an increment of the ring spacing and choosing a different set of seven rings, pressure signals can be measured at different radial distances. The radius at the center of the array is varied in the range from $r / D=1$ to 1.75 . The resultant configuration of the hydrodynamic array for the experiments is illustrated in figure 9. Note that when the array is shifted upstream to measure pressure signals at a larger radius, the first few upstream rings must be removed.

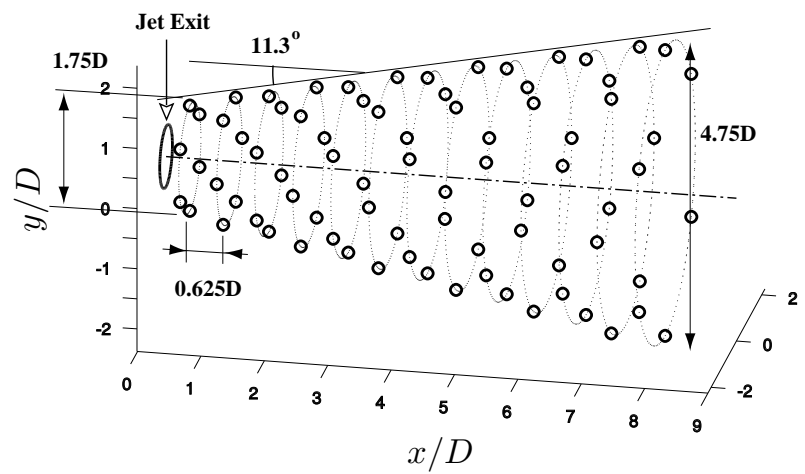

Figure 9: Microphone distribution of the hydrodynamic array designed for high Reynolds number jets.

Thus, the overall microphone array consisted of 13 ring times 6 microphones, a total of 78 quarter-inch microphones. Each microphone was supported by an adopter which was pointing toward the jet axis from a cylindrical steel frame with approximately 1.2 meter diameter and 0.5 meter axial length. This whole array was mounted on a structure with wheels to adjust the axial position of the array. A photograph of the hydrodynamic array set downstream of the jet is displayed in figure 10. The error of the microphone position was estimated within $0.05 \mathrm{D}$ from a test using a single noise source of a spark plug. The back ground noise level was at least three orders of magnitude smaller than signals from a jet in pressure amplitude.

\section{DATA ACQUISITION}

We set the sampling rate of the pressure measurement to be $50 \mathrm{kHz}$ and the band-pass filter to be 20 $\mathrm{kHz}$; thus, for example, the frequency resolution is approximately up to $S t=3$ at $M_{\text {jet }}=0.9$. In the beam-forming as well as other Fourier transforms in time, four periods of the target frequency were processed. Although no windowing function was applied to the segments for a comparison purpose, the uncertainty in amplitude of instability waves due to 


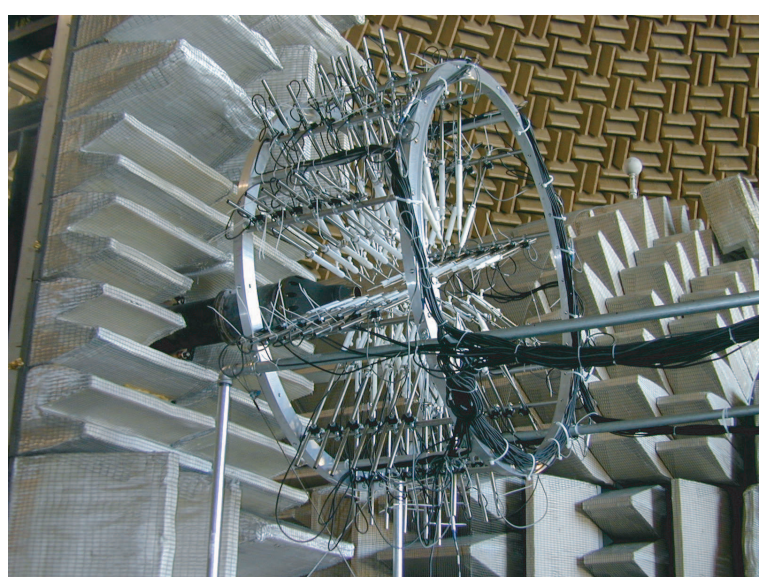

Figure 10: Photograph of the hydrodynamic array set in front of the jet.

windowing is estimated to be less than $3 \%$. For each flow condition, pressure histories were recored for 10 seconds so that at least 200 time series of four periods of the target frequency can be processed. By increasing the number of time series from 200 to 400, the change in amplitude of instability waves is within only $5 \%(\sim 0.4 \mathrm{~dB})$.

\section{RESULTS AND DISCUSSION}

\section{RADIAL DEPENDENCE}

We first identify the region in the radial direction where linear hydrodynamic waves are dominant. As mentioned, for a given flow condition, data were taken with the array shifted to several axial positions to obtain independent data sets at different radii. To evaluate the pressure levels at the radius of each microphone ring, we took Fourier transforms of pressure histories in time and the azimuthal direction at six microphones on each ring and defined it as amplitude.

Figure 11 compares these pressure levels at several radii with eigenfunctions, whose magnitudes are given from the beam-forming. In unheated cases (Cases A and B), the decay rates are well predicted by the eigenfunctions (decaying $\sim r^{-1 / 2}$. $\left.\exp \left[-r \sqrt{k_{x}^{2}-\left(\omega / a_{\infty}\right)^{2}}\right]\right)$ in all three modes at a ring radius smaller than $r / D \leq 1.25$. At a larger radius, acoustic signals become relevant and pressure levels exceed the eigenfunctions. The acoustic signals eventually decay algebraically with a radius $\left(\sim r^{-1}\right)$, which implies a $6 \mathrm{~dB}$ reduction for each doubling of a radius. We can observe that even the most outer radius of the array does not reach the pure acoustic region.

At a higher Mach number (Case $\mathrm{B}, u_{\text {jet }} / a_{\infty}=$ 0.9 ), intensity of the acoustic signals relative to the hydrodynamic disturbances increases for all three modes. As a result, the outer edge of the linear hydrodynamic region shrinks. As the jet velocity increases, the sound intensity follows $\sim U_{\text {jet }}^{8}$ in the far field [16], while the intensity of the pressure fluctuation from hydrodynamic disturbances is proportional to $\sim U_{\text {jet }}^{4}$ according to Bernoulli's equation, assuming that the compressibility effect is small. This indicates, for example, that the pressure level in the near field can increase up to $10 \mathrm{~dB}$ from Case A to Case B, while the sound pressure level can increase as much as $20 \mathrm{~dB}$ in the far field asymptote. The difference between (a) and (b) in figure 11 falls within this range, although it is smaller due to compressibility effects. We discuss this in figure 13 later.

When the jet is heated (Case C), the pressure levels of instability waves increase in the near field due to higher growth rates of instability waves in the streamwise direction. On the other hand, the eigenfunctions decay faster in $r$ compared with the unheated case (Case A). It appears that the pressure levels tend to exceed those given from the beamforming in all three modes. It should be noted that the relative intensity of acoustic signals may be higher in heated jets because the decay of hydrodynamic waves in the radial direction is faster. Moreover, the end of the potential core, which is the primary source of the low frequency noise, is closer to $x / D=2.25$ in the heated jet. These effects may cause the observed discrepancy of the pressure levels.

\section{PHASE CORRELATION}

Next, phase correlation between rings in the streamwise direction is studied. Similar to the data processing in the previous section, phase of the complex amplitude at each ring is computed; subsequently, the averaged phase shifts relative to the fourth ring are plotted and compared with those of eigen-solutions in figure 12. Here, we can confirm our design goal of covering about two wavelengths at $S t=0.35$. At this frequency, the linear stability analysis predicts more rapid phase shift than that of the measurements for all three modes. This implies that the dominant wavelength at this frequency is somewhat longer than the prediction. Yet, we should also emphasize that the beam-forming algorithm can theoretically eliminate modes whose wavenumbers deviate substantially from the target instability modes. Therefore, we expect that the ampli- 
tude obtained from the beam-forming is not significantly influenced by signals other than the target mode.

As the target frequency is decreased to $S t=0.25$, the agreement is substantially improved, although the dominant length-scale of the $m=2$ mode still appears to be longer than the prediction. As figure 2 shows, the most unstable frequency of the $m=2$ mode is lower than the first two modes. Hence, we expect that the agreement of the $m=2$ mode improves at an even lower frequency. From these results, we can deduce that the frequency that can be most accurately captured by the beam-forming decreases as the azimuthal mode number increases. This is consistent with the peak frequencies of the growth rates calculated by the linear stability analysis. In theory, since an eigenfunction is a discrete solution for a given frequency, phase relation should be uniquely determined for each $m$. However, it is possible that signals from neighborhood frequencies or wave-numbers, which have larger magnitude, can lead to contamination.

\section{STREAMWISE EVOLUTION}

Finally, we plot the streamwise evolution of the pressure levels and compared it with eigen-solution, whose amplitude is given from the beam-forming. To be precise, the evolution is plotted along the conical array; hence, the signals appear to saturate and decay somewhat faster than they evolve in the axial direction at constant $r$.

Since the beam-forming algorithm attempts to eliminate signals other than the target instability mode, the estimated amplitude of instability waves should be lower than the pressure level obtained from Fourier transforms. It is possible to eliminate, for example, signals from convecting turbulence at the same frequency but uncorrelated from instability waves, from signal processing. We also should remind that the aliasing error may be contaminated from the higher azimuthal modes. In particular, the raw pressure level in $m=2$ includes the $m=4$ mode, whose relative intensity is higher than that of the contamination in $m=0$ (from $m=6$ ) and $m=1$ (from $m=5$ ). Nonetheless, the proposed algorithm captures streamwise evolution, particularly in Cases $\mathrm{A}$ and $\mathrm{C}$ quite well.

At a higher Mach number (Case B), acoustic signals become more significant downstream; hence, pressure levels keep increasing or reach a plateau, while the eigen-solutions saturate and damp more rapidly. The overall hydrodynamic pressure level is only twice as high as that for Case A. If it follows $\sim U_{\text {jet }}^{4}$, as mentioned before, it must be one order of magnitude higher; thus, the compressibility effects appear to be significant.

In Case $\mathrm{A}$, we notice that the $m=0$ mode grows more rapidly near the nozzle exit compared with the eigenfunction, while the $m=1$ and $m=2$ modes approximately follow the prediction. The linear stability analysis predicts that the growth rate of the $m=0$ mode is much lower than those of the $m=1$ and $m=2$ modes at frequencies lower than the peak (refer to figure 2). However, the actual growth rates of these two modes are comparable in the experiments. Such disagreement is more relevant at $S t=0.25$, as shown in figure 13 (a-ii). This is qualitatively similar to the trend observed in the phase correlation; namely, the beam-forming algorithm agrees better at lower frequencies in higher azimuthal modes. However, the frequency which provides the best agreement in the streamwise evolution seems to be lower than that in phase correlation.

Note that the pressure level in $m=0$ at the first ring in Case B appears much higher than the expected level. This is due to a resonance phenomenon, which may be originated from the internal aerodynamics of the jet. Detailed documentation of this effect is presented in an appendix.

\section{CONCLUSIONS}

We have designed a hydrodynamic array which can measure instability waves in a round jet and evaluated the detection accuracy of the proposed algorithm using a DNS database. We have also tested this technique in experiments. In fact, all three tests, the radial decay, phase correlation, and streamwise evolution, have demonstrated that the linear hydrodynamic region clearly exists even in high Reynolds number jets. The comparison between the measured amplitude and the linear stability analysis exhibits the best agreement near the most amplified frequency of each azimuthal mode.

The results at a lower Mach number (Cases A and C) indicate that the algorithm provides the best prediction near the peak amplitude in the streamwise direction (which is slightly upstream of the end of the potential core). This suggests that hydrodynamic waves can be extracted even when the intensity of instability waves is decreased relative to the acoustic signals at higher Mach numbers. The information of peak amplification is necessary to relate the large-scale coherent structure near the end of the potential core and the far field sound at the peak frequency.

On the other hand, the growth rates of instability waves near the nozzle exit tend to be underestimated, particularly for $m=0$. This may be 
caused by the energy cascade from the most amplified frequency (i.e. nonlinear effects) or associated with the instabilities in the boundary layer inside the jet. In fact, the most unstable frequency of the boundary layer (i.e. Tollmien-Schlichting waves) is estimated to be on the order of $S t \sim O(1)$ or lower, which may interact with the dominant shear-layer instability near the nozzle exit. To study the strategies of active noise control via the modification at the vicinity of the nozzle exit, subtle changes in amplitude of initial instability waves must be detected; therefore, it may be useful to cluster microphone rings near the nozzle exit.

In the future research, we plan to present the data for the chevron nozzles. This will provide the changes of instability wave amplitude due to the nozzle exit modification. It is also of interest to compare the correlation between the instability waves and the far field sound in each azimuthal mode. Some previous experiments and simulations have indicated that the "efficiency" of sound production from the largescale structures is different for different azimuthal modes. Thus, such comparison could provide hints for active noise control.

\section{$\underline{\text { ACKNOWLEDGMENTS }}$}

The authors would like to acknowledge partial support of the AeroAcoustics Research Consortium (AARC) grant from the Ohio Aerospace Institute (OAI). We sincerely appreciate the experiments conducted by Drs. J. Bridges, S.-S. Lee, and their colleagues at NASA Glenn Research Center as well as fruitful discussion with their group. We also thank Prof. J. Freund at the University of Illinois for the DNS database and Drs. S. Narayanan, R. Reba, and R. Schlinker at the United Technologies Research Center for technical discussion regarding jet noise experiments.

\section{References}

[1] V. H. Arakeri, A. Krothapalli, V. Siddavaram, and M. B. Alkislar. On the use of microjets to suppress turbulence in a Mach 0.9 axisymmetric jet. J. Fluid Mech., 490:75-98, 2003.

[2] R. E. A. Arndt, D. F. Long, and M. N. Glauser. The proper orthogonal decomposition of pressure fluctuations surrounding a turbulent jet. J. Fluid Mech., 340:1-33, 1997.
[3] J. P. Bonnet, D. R. Cole, J. Delville, M. N. Glauser, and L. S. Ukeiley. Stochastic estimation and proper orthogonal decomposition: complementary techniques for identifying structure. Exp. Fluids, 17:307-314, 1994.

[4] J. P. Boyd. Complex coordinate methods for hydrodynamic instabilities and Sturm-Liouville eigenproblems with an interior singularity. $J$. Comput. Phys., 57:454-471, 1985.

[5] F. Bretolotti and T. Colonius. On the noise generated by shear-layer instabilities in turbulent jets. AIAA Paper 2003-1062, 2003.

[6] J. Bridges and M. Wernet. Measurements of the aeroacoustic sound source in hot jets. AIAA Paper 2003-3130, 2003.

[7] G. W. Butler and F. T. Calkins. Initial attempts to suppress jet noise using piezoelectric actuators. AIAA Paper 2003-3192, 2003.

[8] B. Callender and E. Gutmark. A near field investigation of chevron nozzle mechanisms. AIAA Paper 2003-3210, 2003.

[9] L. C. Cheung and S. K. Lele. Acoustic radiation from subsonic and supersonic mixing layers with nonlinear PSE. AIAA Paper 2004-0363, 2004.

[10] J. H. Citriniti and W. K. George. Reconstruction of the global velocity field in the axisymmetric mixing layer utilizing the proper orthogonal decomposition. J. Fluid Mech., 418:137166, 2000.

[11] D. G. Crighton and P. Huerre. Shear-layer pressure fluctuations and superdirective acoustic sources. J. Fluid Mech., 220:355-368, 1990.

[12] R. P. Dougherty. Phased array beamforming for aeroacoustics. Lecture Notes in an AIAA Professional Development Short Course, 1999.

[13] R. P. Dougherty and R. W. Stoker. Sidelobe suppression for phased array aeroacoustic measurements. AIAA Paper 98-2242, 1998.

[14] J. B. Freund. Noise sources in a low-Reynoldsnumber turbulent jet at Mach 0.9. J. Fluid Mech., 438:277-305, 2001.

[15] R. A. Gramann and J. W. Mocio. Aeroacoustic measurements in wind tunnels using adaptive beamforming methods. J. Acoust. Soc. Am., 97(6):3694-3701, 1995. 
[16] Lighthill. M. J. On sound generated aerodynamically. 1. General theory. Proc. Roy. Soc. Lond. A., 211:564-587, 1952.

[17] D. H. Johnson and D. E. Dudgeon. Array Signal Processing - Concept and Techniques. PrenticeHall, 1993.

[18] G. M. Lilley. On the noise from jet. In $A G A R D$ CP 131, pages 1-12, 1974.

[19] J. T. C. Liu. Developing large-scale wavelike eddies and the near jet noise field. J. Fluid Mech., 62(3):437-464, 1974.

[20] R. Mankbadi and J. T. C. Liu. A study of the interactions between large-scale coherent structures and fine-grained turbulence in a round jet. Phil. Roy. Soc. Lond., 298:541-602, 1981.

[21] K. Mohseni, T. Colonius, and J. B. Freund. An evaluation of linear instability waves as sources of sound in a supersonic turbulent jet. Phys. Fluids A, 14(10):3593-3600, 2002.

[22] P. J. Morris, M. G. Giridharan, and G. M. Lilley. On the turbulent mixing of compressible free shear layers. Proc. Roy. Soc. Lond. A., 431:219-243, 1990.

[23] M. Mosher. Phased arrays for aeroacoustic testing: Theoretical development. AIAA Paper 961713, 1996.

[24] D. Papamoschou. Noise suppression in moderate-speed multistream jets. AIAA Paper 2002-2557, 2002.

[25] F. J. Piet and G. Elias. Airframe noise source localization using a microphone array. AIAA Paper 97-1643, 1997.

[26] D. C. Pridmore-Brown. Sound propagation in a fluid flowing through an attenuating duct. $J$. Fluid Mech., 4:393-406, 1958.

[27] R. Reba, S. Narayanan, T. Colonius, and M. Dunlop. A study of the role of organized structures in jet noise generation. AIAA Paper 2003-3314, 2003.

[28] M. Samimy, K. B. M. Q. Zaman, and M. F. Reeder. Effect of tabs on the flow and noise field of an axisymmetric jet. AIAA J., 31(4):609619, 1993.

[29] J. C. Simonich, S. Narayanan, T. J. Barber, and M. Nishimura. Aeroacoustic characterization, noise reduction, and dimensional scaling effects of high subsonic jets. AIAA J., 39(11):20622069, 2001.

[30] T. Suzuki and G. W. Butler. New beam-forming algorithm for high speed jet flows. AIAA Paper 2002-2505, 2002.

[31] C. K. W. Tam and P. J. Morris. The radiation of sound by the instability waves of a compressible plane turbulent shear layer. J. Fluid Mech., 98:349-381, 1980.

[32] C. K. W. Tam and K. B. M. Q. Zaman. Subsonic jet noise from nonaxisymmetric and tabbed nozzles. AIAA J., 38(4):592-599, 2000.

[33] H. K. Tanna. Experimental-study of jet noise: 1. Turbulent mixing noise. J. Sound and Vib., 50(3):405-428, 1977.

[34] S. R. Venkatesh, D. R. Polak, and S Narayanan. Beamforming algorithm for distributed source localization and its application to jet noise. AIAA J., 41(7):1238-1246, 2003.

\section{APPENDICES}

\section{EIGENFUNCTION BEYOND NEUTRALLY STABLE POINT}

As Tam \& Morris [31] explained in their appendix, we must carefully consider a branch-cut in the complex $r$ plane when we compute stable or neutrally stable eigenfunctions. As the velocity profile spreads downstream, the growth rate of eigenfunctions vanishes, i.e. $\operatorname{Im}[k]=0$, when $\omega-k U=0$ at some $r$. This point is referred to as a 'critical layer,' corresponding to a branch point in the complex $r$ plane. As the growth rate goes negative further downstream, the imaginary part of the critical point in $r$ becomes positive. To circumvent this branch point, we integrate (8) along

$$
r(\rho)=\rho+\mathrm{i} \Delta \exp \left[-\frac{\left(\rho-\operatorname{Re}\left[r_{*}\right]\right)^{2}}{\sigma^{2}}\right],
$$

where $r_{*}$ satisfies $\omega-k U\left(r_{*}\right)=0$. In this study, we use $\Delta / D=0.04+\operatorname{Im}\left[r_{*}\right]$ and $\sigma / D=0.02$ and integrate (8) in $0 \leq \rho \leq 3 D$ by a shooting method (c.f. Ref. [4]).

\section{FREQUENCY SPECTRUM AT HIGH MACH NUMBERS}

In this experiment, we have observed a resonance phenomenon, particularly at higher Mach numbers 
for the unheated jet. Figure 14 depicts frequency spectra of the first seven rings from the nozzle exit (the data are Fourier decomposed in the azimuthal direction) for Case B. In the $m=0$ mode, the highest peak appears at $S t \approx 0.35$, and their harmonics seem to follow at higher frequencies (although their spacings are not exactly harmonics). These peaks are smeared downstream. In the $m=1$ mode, the spectrum is first peaked at $S t \approx 0.6$, and the qualitative characteristics are similar to those of the $m=0$ mode. Note that the first peak frequency of the $m=2$ mode (not shown) is nearly the same as that of the second peak of the $m=0$ mode. Thus, the first few rings are influenced in the $m=0$ mode in the frequency range of our interests $(S t=0.25 \sim 0.35)$. Because six microphones at the same axial station were not directly tied in the array, this phenomenon is unlikely caused by structural vibration.

One hypothesis is that this sound is originated from the internal aerodynamics. Here, we compute duct acoustic modes in a quiescent space assuming that the wavelength in the axial direction is much longer than the rig radius $(7.62 \mathrm{~cm})$ and denote their Strouhal numbers by arrows in figure 14 . The higher modes correspond to the harmonics in the radial direction (i.e. the Bessel function). Although the first mode does not appear in all cases (it is also the case for $m=2$ ), the duct acoustic modes seem to scale the peak frequencies of this phenomenon. Thus, the agreement partially supports our hypothesis. This tonal sound is alleviated at lower Mach numbers and nearly scaled by the Helmholtz number. However, it is completely absent in heated jets for unknown reasons; hence, it must be further investigated to fully understand this phenomenon.
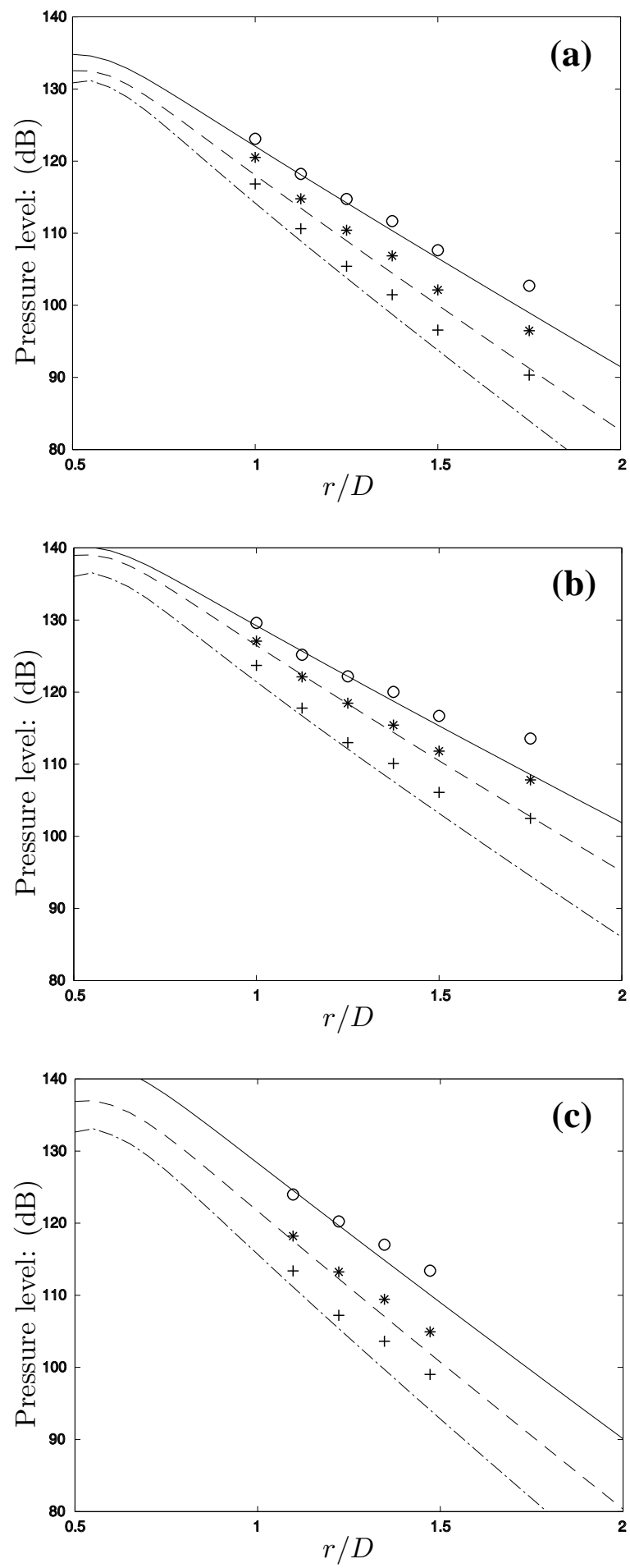

Figure 11: Pressure levels at several radial microphone positions. Pressure levels detected using the beam-forming (i.e. eigenfunction):,$- m=0$; $-{ }_{-}, m=1 ;-\cdot-\cdot-, m=2$. Pressure fluctuation (Fourier transformed in time and the azimuthal direction): $\circ, m=0 ; *, m=1 ;+, m=2$. (a) Case A; (b) Case B; (c) Case C. The target frequency is $S t=0.35$ for all cases. Values are obtained by interpolation at the cross section of $x / D=2.25$. 

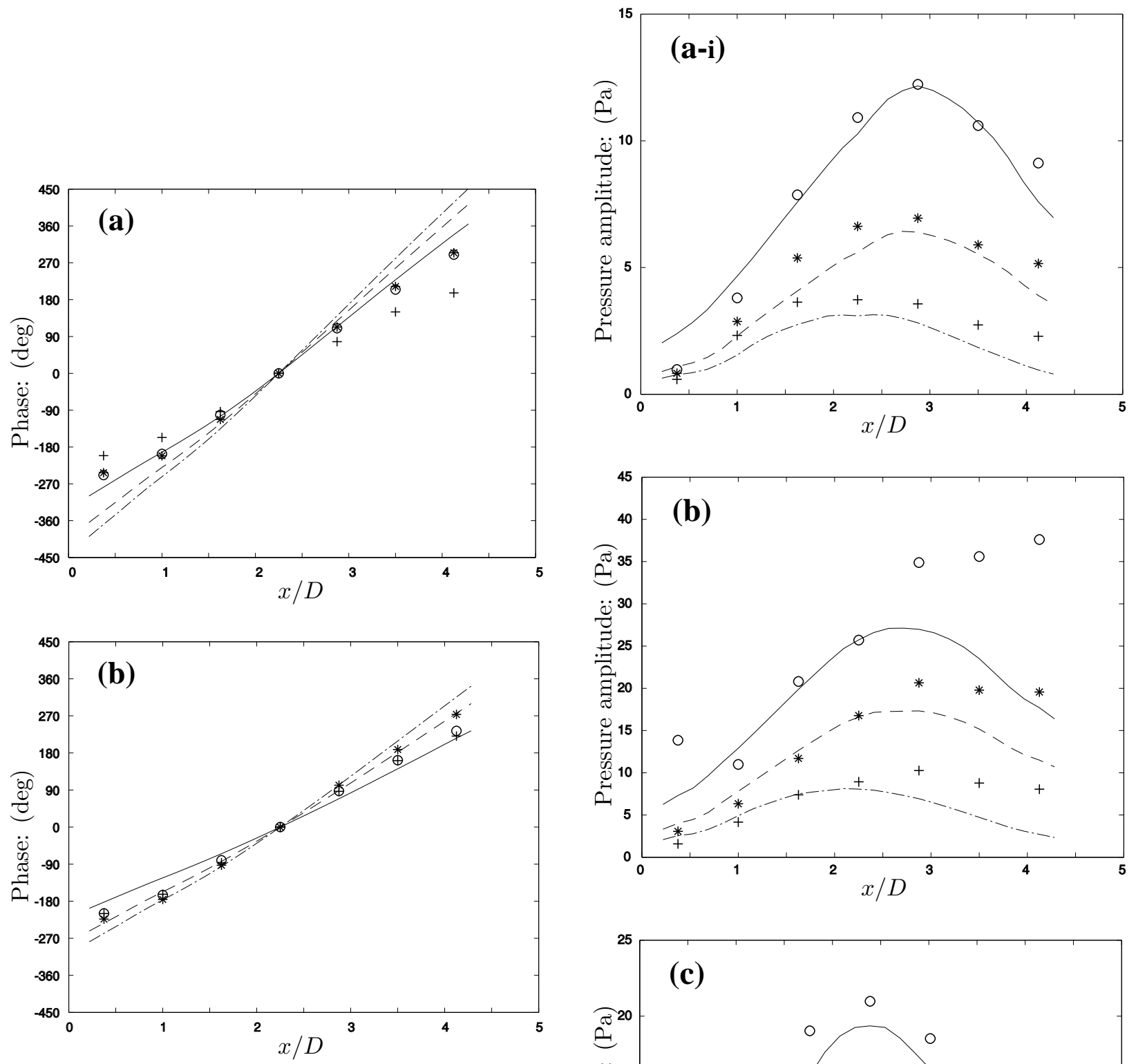

Figure 12: Averaged phase correlation. Phase shifts relative to the fourth ring are plotted: (a), St $=$ 0.35 ; (b), $S t=0.25$. Notation is the same as figure 11.

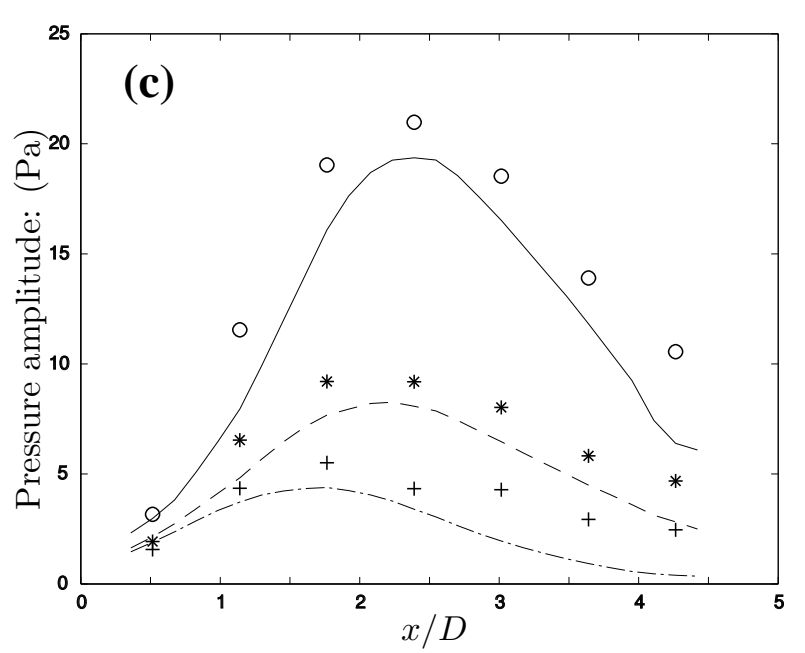




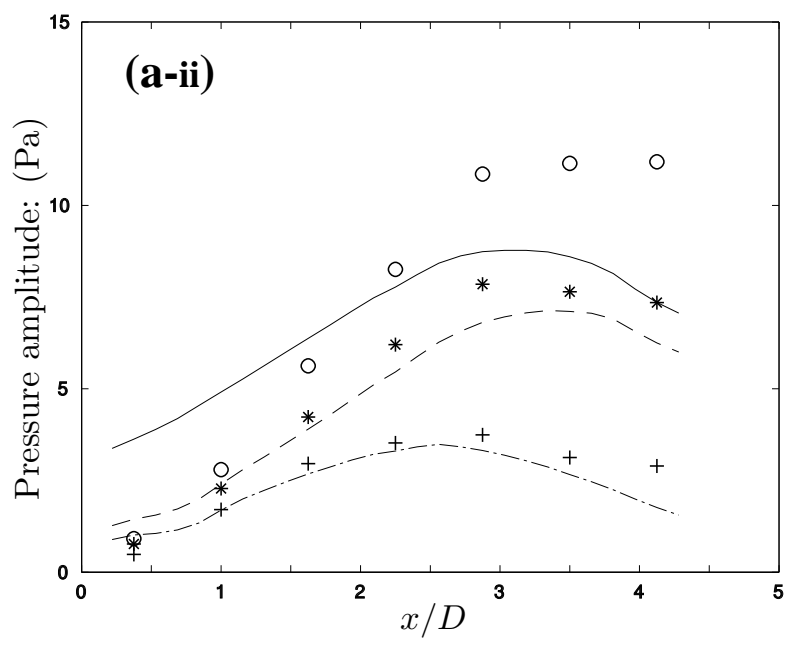

Figure 13: Evolution of pressure amplitude along the microphone array: (a-i) Case A; (b) Case B; (c) Case C; at $S t=0.35$ for these three cases; (a-ii) Case A at $S t=0.25$. Notation is the same as figure 11.
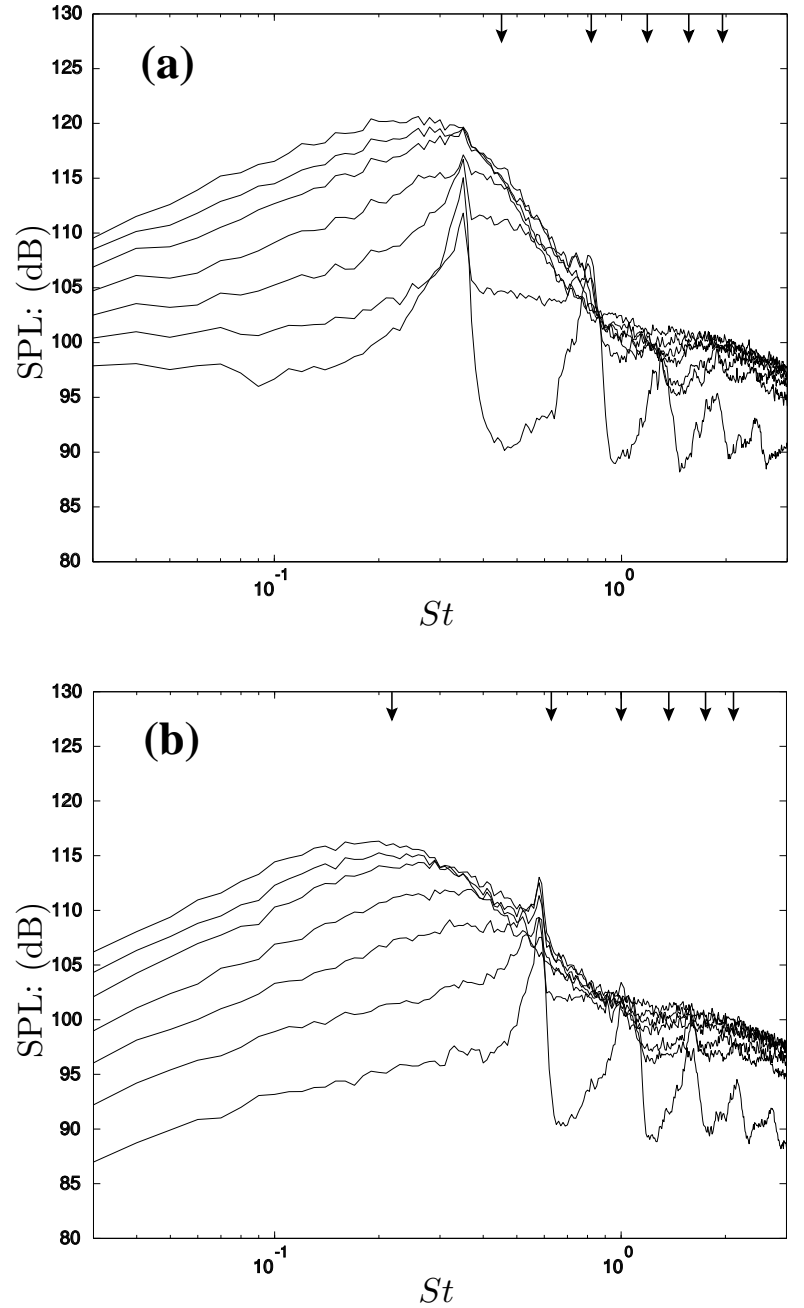

Figure 14: Frequency spectra in Case B. Sound pressure levels of the first seven rings are plotted from the bottom: (a), $m=0$; (b), $m=1$. The arrows denote duct acoustic-mode frequencies in a quiescent flow. 